\title{
Roughness Method to Estimate Fractal Dimension
}

\author{
A. BŁachowski and K. Ruebenbauer* \\ Mössbauer Spectroscopy Division, Institute of Physics, Pedagogical University \\ Podchorążych 2, 30-084 Kraków, Poland
}

(Received January 30, 2009; revised version February 3, 2009)

\begin{abstract}
A method based on the pattern roughness was introduced for determination of the fractal dimension and tested for fractals like the Sierpiński carpet, the Sierpiński triangle, standard Cantor set, the Menger sponge and the Sierpiński tetrahedron. It was tested for non-fractal pattern like two- and four-dimensional gray scale random dust as well. It was found that for all these patterns the Hausdorff dimension is reproduced with relatively high accuracy. Roughness method is based on simple, fast and easy to implement algorithm applicable in any topological dimension. It is particularly suited for patterns being composed of the hierarchy of structures having the same topological dimension as the space embedding them. It is applicable to "fuzzy" patterns with overlapping structures, where other methods are useless. It is designed for pixelized structures, the latter structures resulting as typical experimental data sets.
\end{abstract}

PACS numbers: 05.45.Df, 61.43.Hv

\section{Introduction}

Usually fractal dimension of the "classical" fractals could be expressed in some analytical form and calculated with any required accuracy [1]. The situation is quite different for natural objects approximating some fractal structures. Similar statement applies to the computer-generated structures, the latter structures being similar rather to the natural objects instead of being similar to the "classical" fractals. Many methods have been invented to estimate fractal dimension of such objects [2-5]. It seems that none of them is general, as the successful choice of method depends on details of the fractal in question. We are going to concentrate here on fractals composed of the hierarchy of structures having the same topological dimension as the space needed to embed given fractal. Such structures are quite common, and on the other hand, there are many "classical" fractals having above properties as described in Ref. [1]. The word "classical" is further used as shortened notation for non-random fractals being composed of the objects (hierarchy of structures) having the same topological dimension as the hosting space and being exactly self-similar on all scales.

The most popular method used to estimate fractal dimension for such objects is the box counting method [2]. However, this method has some limitations. It is very hard to apply to the objects having "fuzzy" boundaries, and it is practically useless in the case of overlapping

* corresponding author; e-mail: sfrueben@cyf-kr.edu.pl structures. Overlapping structures are quite common within the realm of natural objects, and fuzziness is always encountered for sufficiently large magnifications of natural objects due to the limited resolution of the imaging devices and real fuzziness of the natural objects.

We are proposing a method to estimate fractal dimension of such objects as mentioned above. It is based on the simple concept of the object roughness seen at various scales. The algorithm is simple, fast and widely applicable to the hierarchical structures having the same topological dimension as the space embedding them. Somewhat similar approach to this problem has been suggested by Zubimendi et al. [5]. Mandelbrot [1] suggested similar idea as well. Roughness could have various meanings and hence we are going to define precisely roughness in the sense used within this contribution. A relation between roughness and fractal dimension has been discussed previously by Mandelbrot [6].

The paper is organized as follows: Sect. 2 deals with the definition of the roughness method as proposed here. Section 3 is devoted to tests performed on the "classical" fractals composed of the hierarchical structures in the sense above defined. Finally, conclusions are drawn in the last Sect. 4.

\section{Roughness method}

Generally, roughness could be defined in any metric Euclidean space having finite topological dimension $D=1,2, \ldots$ and particularly for functions taking on integer values from the range $\left[0 ; 2^{L}-1\right]$, where $L=1,2, \ldots$ stands for the number of bits describing content of the 
volume element called further pixel for brevity. Practically, all experimental or computer generated functions could be transformed into such representation as defined above. All volume elements are identical (they have the same number of bits with the same meaning of each bit for all pixels) and they form hypercubes with all edges mutually orthogonal and of the same length. Hypercubes fill all the space available and do not overlap one with another. There are $N_{\nu}$ hypercubes along each $\nu$-th axis aligned with one of the edges. Index $\nu=1,2, \ldots, D$ enumerates uniquely particular dimension. Each hypercube has $2 D$ nearest neighbors except hypercubes lying on the border of the considered part of the space. Hence, roughness could be expressed as follows:

$$
\begin{aligned}
R & =\left[2 D \prod_{\nu=1}^{D}\left(N_{\nu}-2\right)\right]^{-1} \\
& \times \sum_{\nu=1}^{D} \sum_{n_{\nu}=2}^{N_{\nu}-1} \sum_{k= \pm 1}\left(\frac{x_{n_{\nu}}-x_{n_{\nu}+k}}{2^{L}-1}\right)^{2} .
\end{aligned}
$$

The following condition has to be satisfied: $N_{\nu} \geq 3$. Index $n_{\nu}$ enumerates subsequent hypercubes along the $\nu$-th axis. On the other hand, the pixel content described by integer has to satisfy conditions $0 \leq x_{n_{\nu}} \leq 2^{L}-1$ and $0 \leq x_{n_{\nu}+k} \leq 2^{L}-1$. One can simplify the above definition to the similar form:

$$
\begin{aligned}
\mathrm{R} & =\left[D \prod_{\nu=1}^{D}\left(N_{\nu}-1\right)\right]^{-1} \\
& \times \sum_{\nu=1}^{D} \sum_{n_{\nu}=1}^{N_{\nu}-1}\left(\frac{x_{n_{\nu}}-x_{n_{\nu}+1}}{2^{L}-1}\right)^{2} .
\end{aligned}
$$

For the last definition algorithm is generally faster and it is sufficient to have $N_{\nu} \geq 2$. Condition $0 \leq x_{n_{\nu}+1} \leq$ $2^{L}-1$ has to be satisfied as well. Actually, for $D=1$ and $N_{1} \geq 3$ one has $\mathrm{R} \equiv R$. Nearest neighbor hypercubes have common "walls", the latter having $D-1$ topological dimension. Both definitions lead to the real roughness taking value from the range $[0 ; 1]$. Number of bits used to calculate roughness amounts to

$$
N_{\mathrm{b}}=L \prod_{\nu=1}^{D} N_{\nu} .
$$

Many experimental data sets satisfy above conditions and therefore roughness could be calculated in a straightforward manner for them.

In order to use roughness $R$ to estimate "fractal" dimension one needs series of patterns having all the same sets of $N_{\nu} \geq 3$ parameters and the same constant $L \geq 1$, albeit obtained at vastly different scales, i.e., having all different pixel edge $r_{\lambda}>0$ size. Here the index $\lambda=1,2, \ldots, \Lambda$ enumerates subsequent patterns and the number of patterns satisfies the condition $\Lambda \geq 3$. Subsequent pixel edge sizes have to satisfy the following condition: $0<r_{1}<r_{2}<\ldots<r_{\Lambda}$. One can calculate roughness $R_{\lambda}$ for each pattern and plot $Y_{\lambda}=\ln \left(R_{\lambda}\right)$ versus $X_{\lambda}=\ln \left(r_{\lambda} / r_{1}\right)$ provided $R_{\lambda}>0$ for each pattern $\lambda$.
If one obtains straight line $Y_{\lambda}=\beta+\alpha X_{\lambda}$ one can calculate "fractal" dimension as $d=D-|\alpha|$ provided $|\alpha| \leq D$ $[1,7]$. Actually, the choice of the logarithm base has no effect on the parameter $\alpha$ provided it is positive and the same for $X_{\lambda}$ and $Y_{\lambda}$. One can multiply ratio $r_{\lambda} / r_{1}$ by any arbitrary positive and constant number without affecting $\alpha$ as well. Hence, the abscissa of the above plot could be chosen as the pixel edge size $r>0$ provided the logarithmic scale is used with any positive logarithm base. Multiplication of roughness by the positive constant (the same for all patterns) has no effect on the slope parameter $\alpha$ as well. Plots described above are often called the Richardson plots [1]. Other similar definitions of roughness could be used as well as they lead to the same slope of the Richardson plot, and the slope is the sole relevant parameter to be determined. However, other definitions require more complex algorithms.

The above algorithm is simple, fast and it could be applied to the majority of the experimental data sets. In particular, it could be applied to the "fuzzy" patterns, where other methods like the box counting method are useless. This algorithm is applicable to patterns with overlapping structures as well, where other methods simply fail.

\section{Some tests of the roughness method}

It is essential to test the above hypothesis for the usefulness. It has to produce linear Richardson plots with the correct slopes for the fractal structures. An attempt to perform rigorous proof is rather useless as the method is intended to be applied to the experimental patterns, the latter having none rigorous definition. The simplest way to perform such tests is to apply the roughness method to the fractals having well known fractal dimension in the Hausdorff sense [1]. One has to bear in mind that "classical" fractals (as defined above) are exactly self-similar on all scales, and therefore they do not exist as real objects as nature is not invariant versus arbitrarily large changes of scale. The same statement applies to any computer-generated pattern. Hence, one has to start from some smallest scale by definition of the "elementary cell", the latter being no longer divisible according to the particular fractal algorithm. In fact, natural objects have also small-scale limit due to the fact that matter is composed of atoms. For some fractals like the Sierpiński triangle one has to make suitable approximation while defining "elementary cell" in order to fit into the pixel scheme. It has to be remembered that real data are always pixelized in some way, and hence the pixel scheme is enforced on them at some scale without will of the experimenter. Afterwards, one has to generate particular fractal pattern according to the rules characteristic of the fractal in question. This iterative process has some limit due to the computer limitations as well. Natural objects have similar limits, as they cannot be made arbitrarily large. Usually "classical" fractals have natural scale factor embedded in the generation algorithm. For example, 
standard Cantor set and the Sierpiński carpet require increase in the size along each dimension by factor three in each iterative step, while the Sierpiński triangle has this factor equal to two. Random dust has none natural scale factor and it has no fractal properties, but it is characterized by null "fractal" dimension as it is composed of independent points. For such patterns like random dust it is desirable to apply factor two in order to get as many points as possible within limits of the scale range and without violating of the pixel scheme. Number of accessible scale levels strongly depends on the topological dimension required to generate particular fractal. For large dimensions data volume increases very rapidly in each iteration step.

Once the required pattern has been generated, one has to make respective series of patterns in order to calculate roughness for each of them. Such patterns could be made averaging content of the adjacent pixels in accordance with the particular scale factor, rounding resulting average to the nearest integer and generating new pixel replacing group of the previous pixels. The number of pixels in each dimension is reduced by the scale factor in each step of this iterative procedure until pattern is too small to be used to calculate roughness. Each new pixel is centered on the center of the previous group. Such procedure simulates reduction of magnification for the real patterns as each pixel of the next generation has edge size increased by the scale factor in comparison with the edge size of the pixel belonging to the previous generation. For series of patterns generated this way one can define equivalent abscissa as $S=N /\left(F^{l}\right)$ provided original pattern has the same number of pixels in all dimensions. The symbol $N \gg 0$ stands for the number of pixels, along one of the dimensions, for the original pattern, $F>0$ denotes the scale factor (isotropic here), and $l=0,1, \ldots$ enumerates subsequent iterations leading to the averaged patterns. For random dust and the Sierpiński triangle $F=2$, while for the Sierpiński carpet and standard Cantor set $F=3$. The scale factor $F=3$ applies to the Menger sponge too, while for the Sierpiński tetrahedron one has $F=2$. A logarithmic scale has to be used for this abscissa as well. A pixel belonging to the next generation is an average of $F^{D}$ pixels of the previous generation.

We have performed tests setting $L=8$ ( 8 bits -256 levels) for all patterns investigated. Three patterns with $D=2$ (planar), one pattern with $D=1$ (linear), and two with $D=3$ (flat Euclidean space) have been investigated. For the two-dimensional random dust and the Sierpiński triangle $N=N_{1}=N_{2}=2048=2^{11}$ has been used for the original patterns. In the case of the Sierpiński carpet $N=N_{1}=N_{2}=2187=3^{7}$ has been set for the original pattern, while for the one-dimensional standard Cantor set $N=N_{1}=177147=3^{11}$ has been applied to the original pattern. For the Menger sponge $N=N_{1}=N_{2}=N_{3}=729=3^{6}$ has been applied to the original pattern. The Sierpiński tetrahedron was evaluated setting $N=N_{1}=N_{2}=N_{3}=512=2^{9}$.
Four-dimensional random dust has been treated using $N=N_{1}=N_{2}=N_{3}=N_{4}=128=2^{7}$. "Elementary cells" for the Sierpiński carpet, standard Cantor set and the Menger sponge have been made of black (0) and white (255) elements in the case of the original patterns. Original random dust pattern has been generated using random number generator with the flat distribution and producing integers in the range $[0 ; 255]$. The "elementary cell" of the original pattern approximating the Sierpiński triangle is made of four pixels. Lower pixels have the gray scale (64), while the upper pixels have the gray scale (191). This is the best approximation to the original "elementary triangle" (black) fitting into the white square made of four pixels. Similar problem is encountered for the Sierpiński tetrahedron. The "elementary cell" consists here of eight pixels. Two lower pixels have the gray scale (148), another pair of lower pixels has the gray scale (213), while the four upper pixels split into two pairs having the gray scale (240) and (254), respectively.

It is interesting to note that roughness equals unity for the pattern made of the black and white hypercubes, the latter ordered in the generalized chessboard-like fashion. However, the first averaging with the scale factor equal to two leads to the pattern having roughness equal to zero, and further averaging does not change this roughness anymore. The smallest possible chessboard-like pattern useful to calculate the roughness $R$ in two dimensions is shown in Fig. 1 as a fragment of the larger pattern.

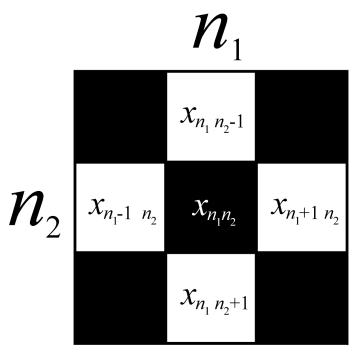

Fig. 1. Black (0) and white (255) chessboard-like pattern (planar), the latter pattern useful for calculation of the roughness $R$.

Generated original patterns for two-dimensional cases are shown in Figs. 2 and 3 together with the corresponding Richardson plots, the latter plots showing $R$ versus $S$. The Richardson plot for the standard Cantor set is shown in Fig. 4. On the other hand, the Richardson plot of the Menger sponge is shown in Fig. 5. The "elementary cell" transformation and the Richardson plot for the Sierpiński tetrahedron is shown in Fig. 6. Finally, the Richardson plot for the four-dimensional random dust is shown in Fig. 7. One has to note that the linear behavior of the Richardson plots is well obeyed except for the largest pixels (smallest magnifications), where blurring is strong enough to almost erase any details of the pattern. These points lying in the lower left corners of the respective plots are excluded and they are not shown. For the Sierpiński triangle one observes some deviation from lin- 

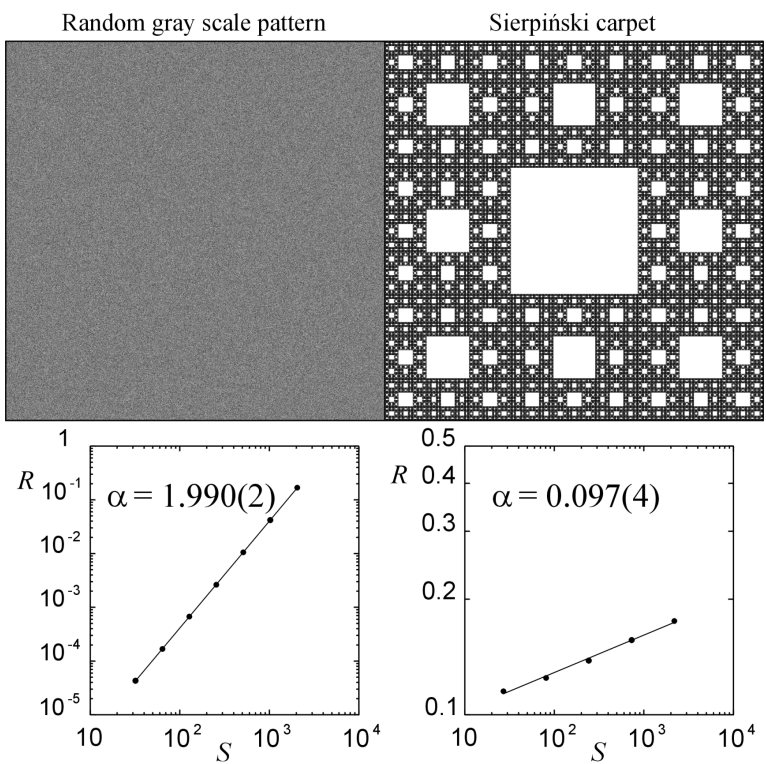

Fig. 2. Original random dust of the gray scale with $D=2$ and the Sierpiński carpet patterns (top) with the corresponding Richardson plots (bottom).

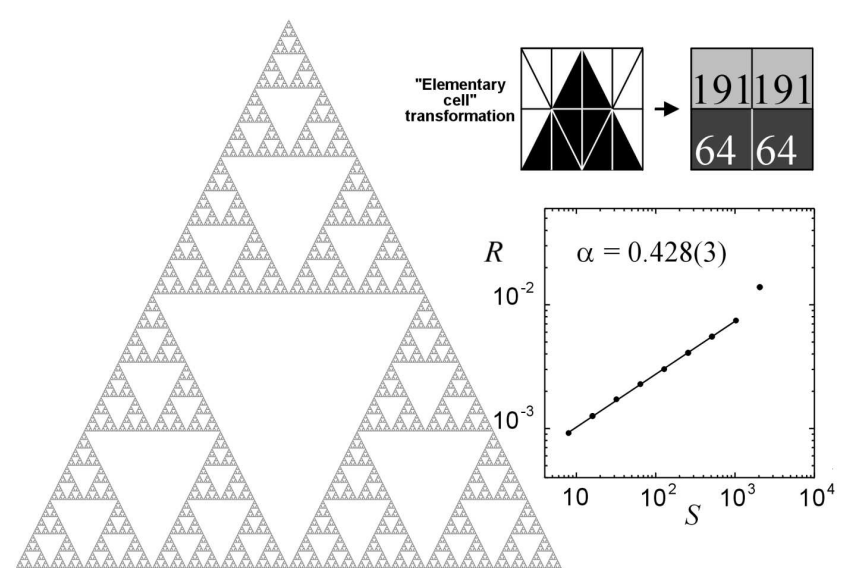

Fig. 3. Original Sierpiński triangle pattern (left) and Richardson plot obtained (right). Inset in the upper right corner shows approximation used for the "elementary cell". Let us note deviation from linearity due to the approximation made while generating the "elementary cell".

earity for the original pattern due to the approximation used to fit pattern into the pixel scheme. The same behavior is seen for the Sierpiński tetrahedron. This point lying in the upper right corner is shown, albeit it has been excluded from the data analysis for both of these fractals. Resulting slope parameters and fractal dimensions are listed in Table. These parameters were obtained by means of the standard linear regression fits to the linear part of the respective Richardson plots. Two-dimensional (planar) patterns are particularly important, as they usually represent some images.

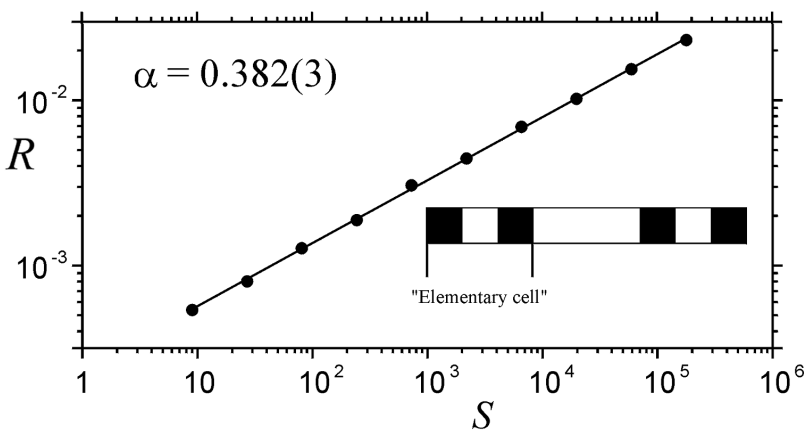

Fig. 4. Richardson plot obtained for the standard Cantor set. Inset shows "elementary cell" and the first step of the iterative procedure used to generate the original pattern.

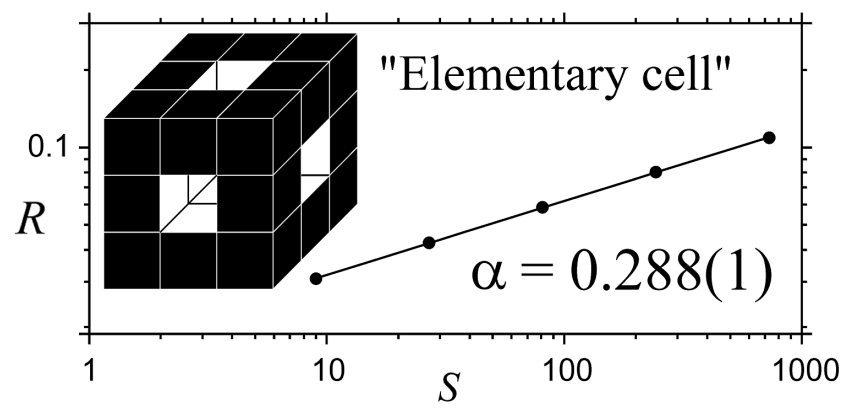

Fig. 5. Richardson plot obtained for the Menger sponge. Inset shows "elementary cell" of this three-dimensional structure. White pixels (255) are shown here as transparent.

Simulations described above show that the roughness method reproduces fractal dimension of the typical fractals with the reasonable accuracy despite limited range of the scale. On the other hand, it is the simplest, most efficient and most widely applicable method in comparison

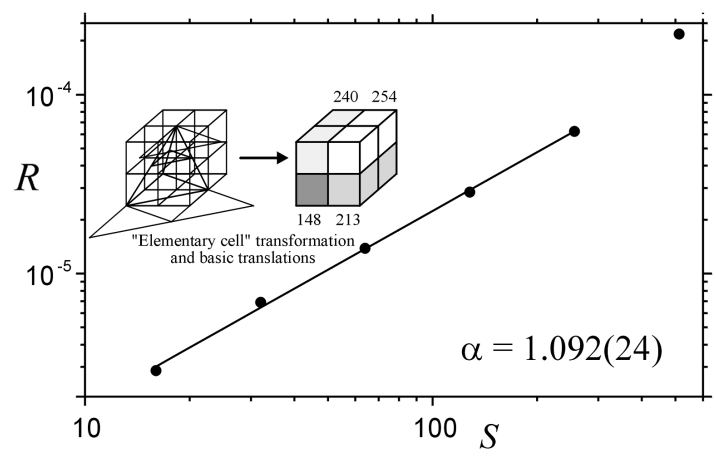

Fig. 6. Richardson plot obtained for the Sierpiński tetrahedron. Inset shows "elementary cell" of this three-dimensional structure and the best approximation of the "elementary cell" within the pixel scheme. The first iterative step is shown schematically as well. Let us note deviation from linearity due to this approximation. 


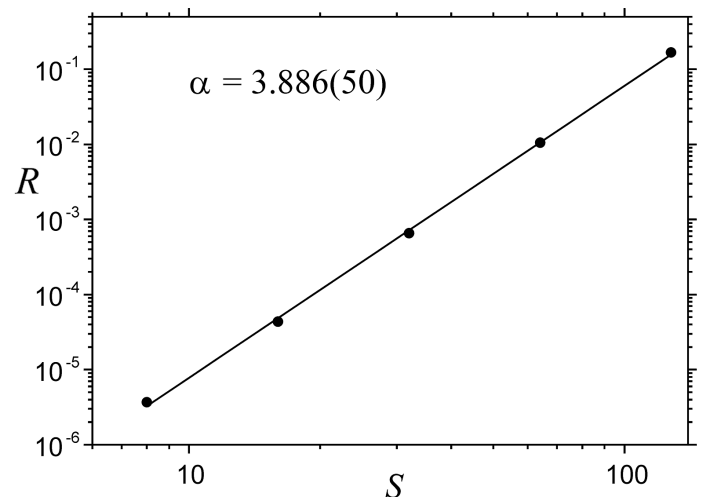

Fig. 7. Richardson plot obtained for the four-dimensional random dust of the gray scale.

TABLE

Fractal dimension obtained by the roughness method in comparison with the corresponding Hausdorff dimension shown with the precision of four digits beyond the decimal point where applicable.

\begin{tabular}{c|c|c|c}
\hline \hline Fractal & $\alpha$ & $\begin{array}{c}\text { Roughness } \\
\text { dimension }\end{array}$ & $\begin{array}{c}\text { Hausdorff } \\
\text { dimension }\end{array}$ \\
\hline $\begin{array}{c}\text { random dust } \\
\text { (non-fractal) } D=2\end{array}$ & $1.990(2)$ & $0.010(2)$ & 0 \\
Sierpiński carpet & $0.097(4)$ & $1.903(4)$ & 1.8928 \\
Sierpiński triangle & $0.428(3)$ & $1.572(3)$ & 1.5850 \\
standard Cantor set & $0.382(3)$ & $0.618(3)$ & 0.6309 \\
Menger sponge & $0.288(1)$ & $2.712(1)$ & 2.7268 \\
Sierpiński tetrahedron & $1.092(24)$ & $1.908(24)$ & 2 \\
random dust & $3.886(50)$ & $0.114(50)$ & 0 \\
(non-fractal) $D=4$ & & &
\end{tabular}

with other classical methods used to estimate fractal dimension. This method has not been reported previously to our best knowledge as applicable to the fractal dimension problem - at least in the form outlined above. It is sensitive to the departure from the ideal pattern as one can see deviations from linearity either for the strongly blurred patterns or upon having applied approximations to the "exact" structure. It could be used to estimate dimension of the non-fractal patterns like the random dust gray scale pattern as well. One has to note that the Sierpiński tetrahedron is somewhat special fractal as it has fractal dimension equal to the topological dimension of the embedded space (plane).

\section{Conclusions}

A method for estimation of the fractal dimension has been proposed. It is based on the simple, fast and widely applicable algorithm, the latter relying on the simple concept of the pattern roughness. It has been tested for typical fractals being a hierarchy of embedded structures with the same topological dimension as the hosting space topological dimension. The Hausdorff dimension has been reproduced with relatively high accuracy for these fractals regardless of the topological dimension. It seems that this method could be particularly useful for the analysis of the two-dimensional gray scale patterns like e.g. electron scanning microscope images. It has been already applied to the analysis of the phase separation in the iron-gold alloys as described in Refs. [8, 9]. For experimental patterns one can estimate fractal dimension by making the Richardson plots of the roughness versus homogeneous scale (the same pixel size along each of the main axes), the latter scale varying from one to another image of the same object. There is no need to have the same number of pixels along each main axis, but these numbers should not differ vastly.

\section{References}

[1] B.B. Mandelbrot, The Fractal Geometry of Nature, Freeman, New York 1982.

[2] L.S. Liebovitch, T. Toth, Phys. Lett. A 141, 386 (1989).

[3] P. Grassberger, I. Procaccia, Phys. Rev. Lett. 50, 346 (1983).

[4] C.W. Lung, J. Jiang, E.K. Tian, C.H. Zhang, Phys. Rev. E 60, 5121 (1999).

[5] J.L. Zubimendi, M.E. Vela, R.C. Salvarezza, L. Vázquez, J.M. Vara, A.J. Arvia, Phys. Rev. E 50, 1367 (1994).

[6] B.B. Mandelbrot, A Theory of Roughness, in: Edge Foundations Inc., Ed. J. Brockman: www.edge.org/ 3rd_culture/mandelbrot04/mandelbrot04_index.html .

[7] B.B. Mandelbrot, Phys. Scr. 32, 257 (1985).

[8] A. Błachowski, K. Ruebenbauer, J. Przewoźnik, J. Żukrowski, J. Alloys Comp. 458, 96 (2008).

[9] A. Błachowski, K. Ruebenbauer, A. Rakowska, in: Problems of Modern Techniques in Engineering and Education, Eds. P. Kurtyka, P. Malczewski, K. Mroczka, I. Sulima, IT Monograph, Kraków 2007, p. 161; see also: www.elektron.ap.krakow.pl/ fractal.pdf . 